\title{
Stage I Pancreatic Cancer AJCC v8
}

National Cancer Institute

\section{Source}

National Cancer Institute. Stage I Pancreatic Cancer A/CC v8. NCI Thesaurus. Code C134915.

Stage I includes: IA: (T1, N0, M0); IB: (T2, N0, M0). T1: Tumor measuring $2 \mathrm{~cm}$ or less in greatest dimension. T2: Tumor measuring more than $2 \mathrm{~cm}$ and $4 \mathrm{~cm}$ or less in greatest dimension. N0: No regional lymph node metastasis. MO: No distant metastasis. (AJCC 8th ed.) 